\title{
Potencialidades da epidemiologia baseada em esgoto nas ações da Atenção Primária à Saúde em tempos de pandemia pela COVID-19
}

\author{
Potentialities of wastewater-based epidemiology in the actions of Primary \\ Health Care in times of pandemic by COVID-19 \\ Potencialidades de la epidemiología basada en aguas residuales \\ en las acciones de la Atención Primaria de Salud en tiempos \\ de pandemia por COVID-19
}

Alexandra Fátima Saraiva SOARES ${ }^{(1)}$ Bárbara Caroline Ricci NUNES ${ }^{(2)}$ Flávia Cristina Rodrigues COSTA ${ }^{(3)}$ Luís Fernando de Morais SILVA ${ }^{(3)}$ Luís Paulo Souza e SOUZA ${ }^{(4)}$

Recebido: 18 jun 2020 Revisado: 30 jun 2020 Aceito: 7 jul 2020

Autor de correspondência: Luís Paulo Souza e Souza luis.pauloss@hotmail.com

Conflito de interesses: Os autores declaram não haver nenhum interesse profissional ou pessoal que possa gerar conflito de interesses em relação a este manuscrito.

\footnotetext{
${ }^{(1)}$ Ministério Público do Estado de Minas Gerais - MPMG, Central de Apoio Técnico, Setor de Meio Ambiente, Belo Horizonte, MG, Brasil.

${ }^{(2)}$ Pontifícia Universidade Católica de Minas Gerais - PUC-MG, Departamento de Engenharia Química, Belo Horizonte, MG, Brasil.

(3) Universidade Federal de Minas Gerais - UFMG, Programa de Pós-Graduação em Saneamento, Meio Ambiente e Recursos Hídricos - PPGSMARH, Belo Horizonte, MG, Brasil.

(3) Universidade Federal do Amazonas - UFAM, Instituto de Saúde e Biotecnologia - ISB,

Departamento de Medicina, Coari, AM, Brasil.
}

\section{Resumo}

Com a presença de materiais genéticos do SARS-CoV-2 nas fezes de indivíduos infectados e em amostras de esgotos sanitários, novos desafios surgiram nas discussões sobre a COVID-19, destacando-se a Epidemiologia Baseada em Esgoto. Considerando o papel que a Atenção Primária à Saúde - APS desempenha na redução da incidência da infecção na população adscrita, com impacto direto nos índices de morbimortalidade, e levando em conta os desafios advindos das novas pesquisas de monitoramento dos esgotos, neste artigo, buscou-se discurtir as pontencialidades da Epidemiologia Baseada em Esgoto nos serviços da APS frente à COVID-19 no Brasil. O monitoramento do esgoto poderia subsidiar ações regionalizadas de contenção do vírus na APS, permitindo antecipar a mobilização dos serviços em áreas potencialmente em risco - onde a circulação do vírus seja detectada pelo monitoramento dos esgotos. Pela característica singular de ter o território como núcleo de análise e atuação, com as informações advindas dos esgotos, os serviços da APS poderiam organizar práticas educativas em cada comunidade, considerando as especificidades locais, a fim de potencializar a coordenação do cuidado e seus demais atributos. A Epidemiologia do Esgoto traria contribuições singulares aos serviços da APS, sendo capaz de monitorar tendências espaciais e temporais, produzir resultados em tempo quase-real (por meio de biossensores), gerar informações em escala populacional e determinar a situação da COVID-19 dentro e entre as comunidades. Ademais, demandaria menos gasto público, quando comparado aos testes clínicos. Frente às limitações financeiras e operacionais para a testagem em massa no Brasil, e mesmo com as limitações quanto à cobertura ideal dos serviços de saneamento em todas as cidades, os esgotos 
surgem como mais um ponto a ser considerado neste momento pandêmico, apresentando-se como uma ferramenta que pode fornecer um diagnóstico coletivo nas comunidades, somando no fortalecimento dos sistemas de vigilância sanitária, epidemiológica e ambiental no país.

Descritores: Infecções por Coronavirus; Betacoronavirus; Vigilância Epidemiológica Baseada em Águas Residuárias; Atenção Primária à Saúde; Vigilância em Saúde Pública.

\section{Abstract}

With the presence of genetic materials of SARS-CoV-2 in the feces of infected individuals and in sanitary sewage samples, new challenges arose in the discussions about COVID-19, especially the Wastewater-based Epidemiology. Considering the role that Primary Health Care - PHC plays in reducing the incidence of infection in the enrolled population, with a direct impact on morbidity and mortality rates, and taking into account the challenges arising from new sewage monitoring studies, in this article, we sought to discuss the potentialities of Wastewater-based Epidemiology in PHC services compared to COVID-19 in Brazil. The monitoring of sewage could subsidize regionalized actions to contain the virus in PHC, allowing to anticipate the mobilization of services in areas potentially at risk - where the circulation of the virus is detected by monitoring the sewers. Due to the unique characteristic of having the territory as a nucleus of analysis and performance, with the information from the sewers, the PHC services could organize educational practices in each community, considering the local specificities, in order to enhance the coordination of care and its other attributes. The Wastewater-based Epidemiology would bring unique contributions to PHC services, being able to monitor spatial and temporal trends, produce results in near-real time (through of biosensors), generate information on a population scale and determine the situation of COVID-19 within and between communities. Moreover, it would require less public spending when compared to clinical trials. Given the financial and operational limitations for mass testing in Brazil, and even with the limitations regarding the optimal coverage of sanitation services in all cities, sewage emerges as another point to be considered at this time pandemic, presenting itself as a tool that can provide a collective diagnosis in the communities, adding in the strengthening of health surveillance systems, epidemiological and environmental in the country.

Keywords: Coronavirus Infections; Betacoronavirus; Wastewater-Based Epidemiological Monitoring; Primary Health Care; Public Health Surveillance.

\section{Resumen}

Con la presencia de materiales genéticos de SARS-CoV-2 en las heces de los individuos infectados y en las muestras de aguas residuales sanitarias, surgieron nuevos desafíos en las discusiones sobre COVID-19, especialmente la Epidemiología Basada en Aguas Residuales. Teniendo en cuenta el papel que desempeña la Atención Primaria de Salud - APS en la reducción de la incidencia de infección en la población inscrita, con un impacto directo en las tasas de morbilidad y mortalidad, y teniendo en cuenta los desafíos derivados de los nuevos estudios de monitoreo de aguas residuales, este artículo buscó reflejar los puntos de Epidemiología Basada en Aguas Residuales en los servicios de APS en comparación con COVID-19 en Brasil. El seguimiento de las aguas residuales podría subvencionar las acciones regionalizadas para contener el virus en la salud, lo que permite anticipar la movilización de servicios en zonas potencialmente de riesgo, donde la circulación del virus se detecta mediante el seguimiento de las alcantarillas. Debido a la característica única de tener el territorio como núcleo de análisis y rendimiento, con la información de las alcantarillas, los servicios de la salud podrían organizar prácticas educativas en cada comunidad, considerando las especificidades locales, con el fin de mejorar la coordinación de la atención y sus otros atributos. La Epidemiología de las Aguas Residuales aportaría contribuciones únicas a los servicios de PHC, pudiendo monitorear tendencias espaciales y temporales, producir resultados en tiempo casi real (a través de biosensores), generar información a escala de población y determinar la situación de COVID-19 dentro y entre comunidades. Además, requeriría menos gasto público en comparación con los ensayos clínicos. Dadas las limitaciones financieras y operativas para las pruebas en masa en Brasil, e incluso con las limitaciones relativas a la cobertura óptima de los servicios de saneamiento en todas las ciudades, las aguas residuales surgen como otro punto a tener en cuenta en este momento la pandemia, 
presentándose como una herramienta que puede proporcionar un diagnóstico colectivo en las comunidades, sumando el fortalecimiento de los sistemas de vigilancia de la salud, epidemiológico y ambiental en el país.

Palabras-claves: Infecciones por Coronavirus; Betacoronavirus; Monitoreo Epidemiológico Basado en Aguas Residuales; Atención Primaria de Salud; Vigilancia de la Salud Pública.

\section{Introdução}

A COVID-19, causada pelo novo coronavírus SARS-CoV-2, já é considerada um dos maiores desafios do século para a saúde pública mundial. Estima-se que uma em cada seis pessoas infectadas desenvolva sintomas mais severos, necessitando de atendimento hospitalar. A maioria, portanto, apresenta sintomas leves, com a possibilidade de, ainda, algumas serem assintomáticas. ${ }^{1,2}$ Assim, é preciso reconhecer que a Atenção Primária à Saúde - APS tem papel fundamental neste cenário, pois maior parte dos casos suspeitos e confirmados será atendida por ela. Contudo, para que sejam ofertados cuidados seguros e de qualidade neste nível de atenção, são necessários planejamento baseado em dados, reorganização dos serviços a partir das características da epidemia, ações e recursos para o enfrentamento da pandemia em curso. ${ }^{3-5}$

Com a divulgação de estudos reportando a presença de materiais genéticos do SARSCoV-2 nas fezes de indivíduos infectados (sintomáticos e assintomáticos) ${ }^{6-8}$ e em amostras de esgotos sanitários, ${ }^{6,9,10}$ novos desafios surgiram nas discussões sobre a COVID-19, com a Epidemiologia Baseada em Esgoto ganhado destaque neste contexto. ${ }^{9-12}$ Desta forma, considerando o papel que a APS desempenha na redução da incidência da infecção na população adscrita, com impacto direto na diminuição da morbimortalidade, ${ }^{3-5}$ e levando em conta os desafios advindos das novas pesquisas de monitoramento dos esgotos, ${ }^{6-12}$ torna-se importante debater como a Epidemiologia Baseada em Esgoto pode contribuir nos serviços da APS. Assim, neste artigo, discute-se as pontencialidades do monitorameno dos esgotos para os serviços da APS no atual contexto de pandemia pela COVID-19, principalmente no contexto brasileiro.

\section{Desenvolvimento}

A partir dos resultados de alguns estudos internacionais ${ }^{6-8}$ e de pesquisas brasileiras, ${ }^{9-10}$ reportando a presença do SARS-CoV-2 nas fezes e nas águas residuais, a Epidemiologia Baseada em Esgoto surge como alternativa promissora, por permitir a detecção do vírus nos esgotos sanitários, ou seja, em escalas comunitárias. A abordagem da Epidemiologia de Esgoto tem sido usada com sucesso para rastrear e fornecer alertas precoces de surtos de vírus como o da hepatite $\mathrm{A}$, o poliovírus e o norovírus. ${ }^{13} \mathrm{~A}$ técnica tem sido bastante 
desenvolvida nas duas últimas décadas, principalmente na Europa, visando o monitoramento do uso de drogas ilícitas. ${ }^{14}$

No contexto da COVID-19, para ser feita uma correlação quantitativa das amostras de ácido ribonucleico (RNA) do vírus encontradas em esgoto com a escala da doença em nível populacional, faz-se necessário determinar a quantidade de RNA presente nas excretas das pessoas e, a partir da concentração encontrada nas amostras, é possível fazer uma extrapolação para a população, utilizando-se de projeções matemáticas e curvas de tendência. ${ }^{9,15}$ Pesquisadores norte-americanos ${ }^{16}$ da cidade de Tempe, no Arizona (EUA), avaliaram as oportunidades e os desafios do uso dessa ferramenta para enumerar infecções ativas pelo SARS-CoV-2 em nível local e global. Foi realizada uma modelagem matemática, associada à análise computacional, para estimar o número de infectados a partir de dados de concentração de RNA do vírus em esgoto. Alguns dos parâmetros incluídos na modelagem foram: faixa de concentração de RNA esperada nas fezes de pessoas infectadas; valores de geração de efluentes per capita, dados da cinética de decaimento para o novo coronavírus, baseado em biomarcadores; efeito da temperatura média do esgoto na cinética

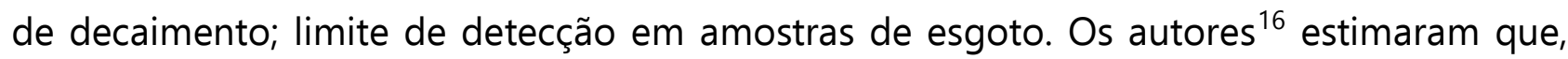
dependendo das condições locais, um caso de infecção (sintomático ou não) poderia ser detectado para a faixa de 100 a 2.000 .000 pessoas não infectadas. Assim, tendo em vista a existência de cerca de 105.600 estações de tratamento de esgotos em operação na cidade do estudo, os autores previram que cerca de 2,1 bilhões de pessoas poderiam ser monitoradas a partir dessa abordagem. ${ }^{16}$

Assim, no contexto brasileiro, o monitoramento dos esgotos pode permitir prever, por exemplo, a velocidade de propagação da doença em determinadas comunidades. $O$ emprego da modelagem ambiental dos esgotos é oportuno para simular e conhecer a contaminação da população, mas vale reforçar que os modelos matemáticos devem ser utilizados de forma complementar e com muita cautela (assim como as modelagens demandam), para que não haja super ou subestimação. ${ }^{16}$

No contexto da APS, a Epidemiologia Baseada em Esgoto poderia subsidiar ações regionalizadas de contenção do vírus, permitindo antecipar a mobilização dos serviços em áreas potencialmente em risco - onde a circulação do vírus seja detectada previamente pelo monitoramento dos esgotos. Além disso, pela característica singular de ter o território como núcleo de análise e atuação, com as informações advindas do monitoramento dos esgotos, os serviços da APS poderiam organizar práticas educativas em cada comunidade ou em microáreas específicas (por meio de campanhas de conscientização e estratégias de testagem - quando possível), considerando as especificidades locais, a fim de potencializar

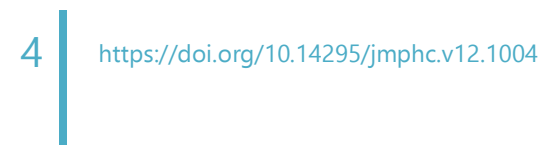


a coordenação do cuidado neste nível de atenção - seus demais atributos ${ }^{3}$ (acesso de primeiro contato, longitudinalidade, integralidade, coordenação do cuidado, competência cultural, e orientação familiar e comunitária).

No Brasil, considerando que é um dos países com maior número de infectados (1.623.284 casos até o dia 06 de julho de 2020 ${ }^{17}$, somada à restrição da testagem em massa e à existência de casos assintomáticos, o monitoramente dos esgotos pode ser instrumento adicional no fortalecimento dos sistemas de vigilância sanitária, epidemiológica e ambiental, ${ }^{11,12}$ principalmente na APS, pois é ela quem recebe $80 \%$ dos casos leves e parte dos casos de média gravidade., ${ }^{3,5}$ Sobre o papel da APS no enfrentamento da COVID-19, autores ${ }^{3}$ debatem:

Apostar naquilo que é a alma da atenção primária, como o conhecimento do território, o acesso, o vínculo entre o usuário e a equipe de saúde, a integralidade da assistência, o monitoramento das famílias vulneráveis e o acompanhamento aos casos suspeitos e leve, é estratégia fundamental tanto para a contenção da pandemia, quanto para o não agravamento das pessoas com a Covid-19., p. 2

Assim, a Epidemiologia do Esgoto traria contribuições singulares aos serviços da APS, sendo capaz de monitorar tendências espaciais e temporais, produzir resultados em tempo quase-real (por meio de biossensores) ${ }^{18}$, gerar informações em escala populacional e determinar a situação da COVID-19 dentro e entre as comunidades.

Destaca-se que, no caso de monitoramento da COVID-19 pelos esgotos, os estudos desenvolvidos até o momento se basearam em amostragens representativas, objetivando monitorar curvas de contágio que podem se estender por semanas, meses ou anos. Assim, autores têm debatido que amostragens conduzidas a cada semana seriam melhores, por permitir otimizar recursos financeiros e logísticos importantes nas análises. ${ }^{18}$

É essencial que as empresas prestadoras dos serviços de saneamento alinhem suas atuações, obtendo controle sobre os sistemas de esgotamento sanitário, para que sejam fornecidos dados de qualidade e capazes de orientar as medidas preventivas de contágio, mitigação dos riscos e cuidados. Assim, um banco de dados e um sistema sentinela poderiam ser criados, para que sejam alimentados com as informações sobre a presença do SARS-CoV-2 nos esgotos por regiões, de forma que todos os serviços das Redes de Atenção à Saúde - RAS tenham conhecimento, buscando uma integração entre eles.

Os cuidados às pessoas em relação à COVID-19 precisam estar presentes nas diferentes fases da infecção e em todo o espectro de gravidade, em uma linha de cuidado que envolva desde a prevenção de novas infecções, monitoramento de casos leves em isolamento domiciliar, identificação precoce dos sinais de agravamento, internação em unidades de terapia intensiva (UTI) e a fase pós alta hospitalar para reabilitação. ${ }^{4}$ Tem sido 
comum observar maior preocupação dos gestores estaduais e municipais quanto aos serviços de pronto atendimento, criação de leitos e hospitais de campanha. Contudo, sem o fortalecimento da Atenção Primária à Saúde, nem sempre é possível garantir que o usuário tenha acesso aos leitos ou aos hospitais de campanha. Por isso, é essencial valorizar os serviços da APS, que, por vezes, ficam esquecido pelas autoridades sanitárias e pela mídia hegemônica no atual contexto de pandemia. Autores ${ }^{5}$ discutem que, embora fortes sistemas de epidemiologia e vigilância sejam ferramentas indispensáveis para a detecção e monitoramento de surtos e emergências de saúde pública, serviços da Atenção Primária à Saúde fortalecidos formam a base de qualquer resposta a emergências.

Ademais, em termos econômicos e práticos, a Epidemiologia Baseada em Esgoto se apresenta em ordens de magnitude mais barato e rápido quando comparada à triagem clínica. ${ }^{11,12,18}$ Autores reforçam que os testes de diagnóstico clínico são inadequados, do ponto de vista financeiro, para monitoramento rápido da incidência da COVID-19 em certas populações, não sendo viável realizar testes individuais repetidos em grandes escalas. ${ }^{16,18}$

A estimativa da presença do SARS-CoV-2 nos esgotos se faz relevante para embasar as decisões das autoridades, especialmente no que concerne ao conhecimento da necessidade de avanço ou redução dos esforços de distanciamento social ou de outras medidas mais rígidas, como o lockdown. ${ }^{12} \mathrm{E}$ a APS tem esta função de ampliar a capacidade de resposta local não só pela redução da infecção, mas, também, pela capacidade de amenizar os efeitos sociais e econômicos das medidas de distanciamento social. ${ }^{4}$ Tal observação reforça mais uma potencialidade da Epidemiologia Baseada em Esgoto nos serviços da APS no contexto brasileiro.

Além disso, tal ferramenta pode subsidiar decisões dos serviços da atenção secundária e terciária, principalmente na alocação de recursos para criação de Unidades de Pronto Atendimento - UPA para os casos de média complexidade e na abertura de novos leitos hospitalares para tratamentos intensivos. ${ }^{12}$

Com a informação de áreas com maior circulação do vírus por meio do monitoramento dos esgotos e das modelagens ambientais, os gestores da Atenção Primária à Saúde poderão direcionar ações que possam interver na ocorrência de novos casos, sem deixar de acompanhar os já instalados e com sintomas leves, além de identificar precocemente os casos graves que necessitam ser manejados para os serviços especializados. De forma complementar e coordenada pela APS, a atenção secundária poderá acompanhar se a circulação do vírus atinge picos muito elevados, ficando em alerta para possíveis casos que procurem o serviço, criando leitos de retaguarda, por exemplo. E, assim, a atenção terciária poderá, também, se organizar quanto à abertura de leitos de 
tratamento intensivo e alocação de recursos humanos. Para isso, torna-se essencial o fortalecimento da coordenação entre todos os níveis assistenciais, com fluxos e pactuações bem definidos.

A partir da Epidemiologia Baseada em Esgoto, os serviços da APS e os dos outros níveis de atenção podem elaborar mapas de contágio, curvas de comunidades infectadas e curvas de assintomáticos, subsidiando a alocação de recursos e profissionais para regiões prioritárias de forma rápida e efetiva.

Apesar das limitações quanto à cobertura ideal do esgotamento sanitário em todas as cidades brasileiras, os esgotos surgem como mais um ponto a ser considerado neste momento pandêmico, apresentando-se como uma ferramenta que pode fornecer um diagnóstico coletivo, especialmente nas maiores cidades que já dispõem de sistema de esgotamento sanitário com coleta que atende percentual acima de 50\% da população. Reconhece-se que os testes clínicos em massa para o diagnóstico da COVID-19 são preferenciais, todavia, no atual contexto de limitações financeiras e operacionais para esta medida no Brasil, a Epidemiologia Baseada em Esgoto representa um complemento, somando no fortalecimento dos sistemas de vigilância sanitária, epidemiológica e ambiental no país.

\section{Conclusões}

A Epidemiologia Baseada em Esgoto é uma ferramenta que precisa ser incorporada nos serviços de vigilância da COVID-19 no Brasil, principalmente no contexto da Atenção Primária à Saúde, pois seria capaz de contribuir de forma singular com os serviços, monitorando tendências espaciais e temporais, gerando informações em escala populacional e determinando a situação da COVID-19 dentro e entre as comunidades, ou seja, fazendo um diagnóstico coletivo. Além disso, seria uma estratégia menos onerosa, demandando menos gastos públicos quando comparada aos testes clínicos. Como o Brasil já apresenta limitações financeiras e operacionais para a testagem em massa, torna-se importante ampliar a discussão desta temática nas ações do sistema de saúde para enfrentamento da pandemia.

\section{Referências}

1. Liu J, Liao X, Qian S, Yuan J, Wang F, Liu Y, et al. Community transmission of severe acute respiratory syndrome coronavirus 2, Shenzhen, China. Emerg Infect Dis 2020;26(6):1320-1323. http://dx.doi.org/10.3201/eid2606.200239. Epub 2020 Jun 17. 
2. Organização Pan-Americana da Saúde - OPAS. Folha informativa - COVID-19 (doença causada pelo novo coronavírus). Brasília, DF: OPAS; 2020 [citado 21 maio 2020].

Disponível em:

http://www.paho.org/bra/index.php?option=com_content $\&$ view $=$ article\&id $=6101:$ covid 19\&Itemid $=875$.

3. Sarti TD, Lazarini WS, Fontenelle LF, Almeida APSC. Qual o papel da Atenção Primária à Saúde diante da pandemia provocada pela COVID-19?. Epidemiol Serv Sade. 2020;29(2):e2020166. http://dx.doi.org/10.5123/s1679-49742020000200024.

4. Daumas RP, Silva GA, Tasca R, Leite IC, Brasil P, Greco DB, et al. O papel da atenção primária na rede de atenção à saúde no Brasil: limites e possibilidades no enfrentamento da COVID-19. Cad Saude Publica. 2020;36(6):e00104120. https://doi.org/10.1590/0102-311X00104120.

5. Dunlop C, Howe A, Li D, Allen LN. The coronavirus outbreak: the central role of primary care in emergency preparedness and response. BJGP Open. 2020;4(1):pii:bjgpopen20X101041. https://doi.org/10.3399/bjgpopen20X101041.

6. Kitajima M, Ahmed W, Bibby K, Carducci A, Gerba CP, Hamilton KA, et al. SARS-CoV-2 in wastewater: State of the knowledge and research needs. Sci Total Environ. 2020;739:139076. https://doi.org/10.1016/j.scitotenv.2020.139076.

7. Zhang Y, Chen $C$, Zhu S, Shu C, Wang D, Song J, et al. Notes from the field: isolation of 2019-nCoV from a stool specimen of a laboratory-confirmed case of the coronavirus disease 2019 (COVID-19). China CDC Wkly 2020;2(8):123-4. http://dx.doi.org/10.46234/ccdcw2020.033.

8. Sun J, Zhu A, Li H, Zheng K, Zhuang Z, Chen Z, et al. Isolation of infectious SARS-CoV-2 from urine of a COVID-19 patient. Emerg Microbes Infect. 2020 Dec;9(1):991-3. http://dx.doi.org/10.1080/22221751.2020.1760144.

9. Universidade Federal de Minas Gerais. Coronavírus é identificado em esgotos [Internet]. Belo Horizonte: UFMG; Belo Horizonte; 06 de maio de 2020 [citado 2020 maio 21]. Disponível em: http://www.ufmg.br/prae/noticias/coronavirus-e-identificado-emamostras-de-esgoto.

10. Ferreira V. Fiocruz divulga estudo sobre a presença do novo coronavírus em esgotos sanitários [Internet]. Rio de Janeiro: Fiocruz; 28 abr. 2020 [citado 2020 maio 21]. Disponível em: http://portal.fiocruz.br/noticia/fiocruz-divulga-estudo-sobre-presencado-novo-coronavirus-em-esgotos-sanitarios. 
11. Souza LPS, Soares AFS, Nunes BCR, Costa FCR, Silva LFM. Presença do novo coronavírus (SARS-CoV-2) nos esgotos sanitários: apontamentos para ações complementares de vigilância à saúde em tempos de pandemia. VD. 2020 jun 2;Ahead of Print. http://dx.doi.org/10.22239/2317-269x.01624

12. Soares AFS, Nunes BCR, Costa FCR, Silva LFM, Souza LPS. Sewage-based epidemiology and covid-19 in Brazil: a necessary discussion. Int J Dev Res. 2020;10(6):36476-82. https://doi.org/10.37118/ijdr.18991.06.2020.

13. Mao K, Zhang H, Yang Z. Can a Paper-Based Device Trace COVID-19 sources with wastewater-based epidemiology?. Environ Sci Technol. 2020;54(7):3733-5. https://doi.org/10.1021/acs.est.0c01174.

14. Choi PM, Tscharkea BJ, Donner E, O'Brien JW, Grant SC, Kaserzon SL, et al. Wastewaterbased epidemiology biomarkers: past, present and future. Trends Analyt Chem. 2018;105:453-69. https://doi.org/10.1016/j.trac.2018.06.004.

15. Mallapaty S. How sewage could reveal true scale of coronavirus outbreak. Nature 2020;580(7802):176-7. https://doi.org/10.1038/d41586-020-00973-x.

16. Hart OE, Halden RU. Computational analysis of SARS-CoV-2/COVID-19 surveillance by wastewater-based epidemiology locally and globally: Feasibility, economy, opportunities and challenges. Sci Total Environ. 2020;730:138875.

https://doi.org/10.1016/j.scitotenv.2020.138875. Epub 2020 Apr 22.

17. Brasil. COVID19 - Painel Coronavírus Brasil [Internet]. Brasília, DF: Ministério da Saúde; 2020 [citado 2020 jul. 6]. Disponível em: https://covid.saude.gov.br/

18. Sodré FF, Brandão CCS, Vizzotto CS, Maldaner AO. Epidemiologia do esgoto como estratégia para monitoramento comunitário, mapeamento de focos emergentes e elaboração de sistemas de alerta rápido para COVID-19. Quim Nova. 2020;43(4):515-9. https://doi.org/10.21577/0100-4042.20170545. 


\section{Minicurrículo}

Alexandra Fátima Saraiva Soares | ORCiD: 0000-0002-9894-627X

Doutora em Saneamento, Meio Ambiente e Recursos Hídricos pela Universidade Federal de Minas Gerais - UFMG, Brasil. Pós-Doutorado em Direito Público pela Università degli Studi di Messina, Itália. Especialista em Direito Sanitário pela Escola de Saúde Pública do Estado de Minas Gerais - ESPMG. Perita Ambiental do Ministério Público do Estado de Minas Gerais - MPMG, Brasil.

Bárbara Caroline Ricci Nunes | ORCiD: 0000-0002-0518-7332

Doutora em Saneamento, Meio Ambiente e Recursos Hídricos pela Universidade Federal de Minas Gerais - UFMG. Professora do Departamento de Engenharia Química da Pontifícia Universidade Católica de Minas Gerais - PUCMG, Brasil.

Flávia Cristina Rodrigues Costa | ORCiD: 0000-0002-9140-7740

Mestranda em Saneamento, Meio Ambiente e Recursos Hídricos pela Universidade Federal de Minas Gerais - UFMG, Brasil.

Luís Fernando de Morais Silva | ORCiD: 0000-0002-3027-1958

Mestrando em Saneamento, Meio Ambiente e Recursos Hídricos pela Universidade Federal de Minas Gerais - UFMG. Engenheiro Trainee no Setor de Meio Ambiente do Ministério Público do Estado de Minas Gerais - MPMG, Brasil.

Luís Paulo Souza e Souza | ORCiD: 0000-0002-9801-4157

Doutor em Saúde Pública pelo Departamento de Medicina Preventiva e Social da Universidade Federal de Minas Gerais - UFMG. Pós-Doutorado em Educação pela Universidade do Estado do Pará - UEPA. Professor Credenciado da Escola de Saúde Pública do Estado de Minas Gerais - ESPMG. Professor Adjunto do Curso de Graduação em Medicina do Instituto de Saúde e Biotecnologia - ISB da Universidade Federal do Amazonas - UFAM, Brasil. 